\title{
A TEORIA DO CUIDADO TRANSPESSOAL NA ENFERMAGEM: ANÁLISE SEGUNDO MELEIS
}

Carlos Magno Carvalho da Silva ${ }^{1}$, Geilsa Soraia Cavalcanti Valente ${ }^{2}$, Graziele Ribeiro Bitencourt ${ }^{3}$, Leonardo Nogueira de Brito $^{4}$

RESUMO: A teoria de Watson defende o cuidado como uma ciência humana desenvolvida a partir de fundamentos filosóficos e sistemas de valores humanistas. Essa perspectiva é baseada em uma relação ontológica por estar atrelada a uma visão mundial de unidade. Destarte, o objetivo deste estudo é descrever a Teoria do Cuidado Transpessoal de Jean Watson com base na etapa da descrição do Modelo de Avaliação de Teorias de Meleis. A metodologia utilizada foi a análise interpretativa com a descrição do modelo especificamente nos componentes estruturais da teoria. Concluímos que o cuidado transpessoal apresenta fundamental valia para o enriquecimento profissional e favorece a recuperação do paciente, pois prioriza a integralidade da atenção, utilizando procedimentos tecnológicos e a consideração de suas necessidades socioculturais e espirituais.

PALAVRAS-CHAVE: Enfermagem; Teoria de enfermagem; Cuidados de enfermagem.

\section{TRANSPERSONAL NURSING CARE THEORY: ANALYSIS ACCORDING TO MELEIS}

ABSTRACT: Watson's theory defends care as a human science developed from philosophical and humanistic value systems. This perspective is based on an ontological relationship by being tied to a vision of world of unity. Thus the aim of this study is to describe Jean Watson's Transpersonal Caring Theory based on the Meleis' Model of Theories' Evaluation step of description. The methodology used was the interpretive analysis describing the specific model in the structural components of the theory. We conclude that transpersonal care has fundamental value for professional enrichment and enhances the patient's recovery, as it focuses on comprehensive care, using technological procedures and consideration of their socio-cultural and spiritual needs.

KEYWORDS: Nursing; Nursing theory; Nursing care.

\section{LA TEORÍA DEL CUIDADO TRANSPERSONAL EN LA ENFERMERÍA: ANÁLISIS SEGÚN MELEIS}

RESUMEN: La teoría de Watson defiende el cuidado como una ciencia humana desarrollada con base en fundamentos filosóficos y sistemas de valores humanistas. Esa perspectiva es basada en una relación ontológica porque está relacionada a una visión mundial de unidad. Así, el objetivo de este estudio fue describir la Teoría del Cuidado Transpersonal de Jean Watson con base en la etapa de la descripción del Modelo de Evaluación de Teorías de Meleis. La metodología utilizada fue el análisis interpretativo con la descripción del modelo específicamente en los componentes estructurales de la teoría. Se concluye que el cuidado transpersonal presenta fundamental importancia para el enriquecimiento profesional y favorece la recuperación del paciente, pues prioriza la integralidad de la atención, utilizando procedimientos tecnológicos y la consideración de sus necesidades socioculturales y espirituales.

PALABRAS CLAVE: Enfermería; Teoría de enfermería; Cuidados de enfermería.

${ }^{1}$ Enfermeiro. Mestrando do Programa de Pós-Graduação em Ciências do Cuidado em Saúde da Escola de Enfermagem Aurora de Afonso Costa, da Universidade Federal Fluminense-EEAAC-UFF.

${ }^{2}$ Enfermeira. Doutora em Enfermagem. Professor Adjunto do Departamento de Fundamentos de Enfermagem e Administração da EEAAC-UFF. Coordenadora do Grupo de Interesse em Pesquisa Educação e Gerência em Enfermagem-GIPEGE-UFF. Membro do Núcleo de Pesquisa Educação e Saúde em Enfermagem-NUPESEnf da Universidade Federal do Rio de Janeiro-UFRJ. Membro do Núcleo de Pesquisa em Cidadania e Gerência em Enfermagem-NECIGEN-UFF

${ }^{3}$ Enfermeira. Pós-graduada em Enfermagem Gerontológica. Professora substituta do Departamento de Enfermagem Médico Cirúrgica da EEAAC-UFF.

${ }^{4}$ Enfermeiro Intensivista do Hospital Estadual Alberto Torres-RJ.

Autora correspondente:

Geilsa Soraia Cavalcanti Valente

Escola de Enfermagem Aurora de Afonso Costa

Rua Dr. Celestino, 74 - 24020-091 - Niteroi-RJ, Brasil

Recebido: 21/12/09

E-mail: geilsavalente@yahoo.com.br

Aprovado: 20/08/10

Cogitare Enferm. 2010 Jul/Set; 15(3):548-51 


\section{INTRODUÇÃO}

O cuidar é visto como a essência da Enfermagem, caracterizado pela reciprocidade entre a equipe de enfermagem e a pessoa cuidada. Destarte, o cuidado pode estar relacionado com a interação entre seres humanos através da troca de subjetividade, consentindo o inter-relacionamento entre quem cuida e é cuidado ${ }^{(1)}$.

Neste contexto, a teoria de enfermagem pode ser definida como "um agrupamento imaginativo do conhecimento, ideias e experiências que são representadas simbolicamente e buscam esclarecer um dado fenômeno"(2:29). Deste modo, têm a finalidade de correlacionar pesquisa e prática, tornando possível a confiabilidade das ideias e experiências dos enfermeiros, como valiosa fonte de conhecimento( ${ }^{(2)}$.

A Teoria do Cuidado Transpessoal de Watson pode ser classificada como interacionista, uma vez que sua prática acontece através das interações paciente/ enfermeira. Esta influência mútua no cuidado é uma experiência que necessita de diálogo entre pessoas, no qual cada uma delas sente a disponibilidade, a proximidade e a compreensão uma da outra, além de partilharem histórias de vida, trajetórias e angústias ${ }^{(3)}$.

Com isso, utilizamos a Teoria do Cuidado Transpessoal de Jean Watson como foco de nosso trabalho, objetivando descrevê-la segundo a etapa de descrição do Modelo de Avaliação de Teorias de Meleis.

A relevância deste estudo está: no ensino, por meio da fundamentação sólida em ciências humanas, as quais podem fornecer embasamento à ciência do cuidado; na prática, através do desenvolvimento de pensamentos críticos inerentes ao exercício da Enfermagem; e na pesquisa, envolvida na busca por investigações do processo do cuidado humano.

\section{MÉTODO}

Trata-se de um estudo de análise interpretativa com base na descrição do Modelo de Avaliação de Teorias proposto por Meleis pelo qual é possível a discussão de componentes funcionais como foco, cliente, enfermagem e ambiente ${ }^{(4)}$.

Este modelo é dividido nos seguintes segmentos: descrição, análise, crítica, teste e apoio. Neste estudo, a ênfase principal está na descrição, já que é uma etapa responsável por identificar elementos conceituais pertinentes às ideias centrais de uma teoria, no caso, a reflexão sobre a Teoria do Cuidado Transpessoal de Jean Watson ${ }^{(5)}$.
Para melhor compreensão, utilizou-se as etapas descritas como subtítulos deste estudo, a fim de favorecer a identificação dos elementos estruturais pertencentes às ideias centrais e a crítica reflexiva sobre a Teoria do Cuidado Transpessoal de Jean Watson $^{(5)}$.

Como questões centrais para a etapa de descrição dos componentes estruturais, pontua-se: a) se a teoria identifica o foco da teoria com o cliente, família, comunidade, sociedade ou não; b) qual a definição que a teoria oferece à Enfermagem, cliente, saúde, problemas de enfermagem, relacionamento enfermeiro-paciente; c) se a teoria oferece uma ideia clara sobre os problemas de enfermagem, e d) se a teoria oferece algum insight no que concerne à intervenção de enfermagem ${ }^{(6)}$.

No estudo em questão, foi enfocada, principalmente, a "etapa b" como proposta para favorecer o entendimento dos componentes estruturais (conceitos, pressupostos e proposições) da teoria em estudo ${ }^{(5)}$.

\section{Descrição da teoria do cuidado tranpessoal}

Trata-se de uma teoria que propõe intervenção consciente nos cuidados, potencializando a cura e a integridade. Não descarta a ciência convencional ou práticas de enfermagem modernas, mas é um complemento às mesmas. Descreve a conscientização voltada ao levantamento de quaisquer questões sobre o que significa cuidar, estar enfermo e ser cuidado/curado. Prioriza a preservação da saúde e procura meios para proteger, melhorar e preservar a dignidade, humanidade, integridade e harmonia interior de uma pessoa ${ }^{(8)}$.

O cuidado transpessoal é um conceito criado pela Doutora em Enfermagem Jean Watson, o qual desvia o foco da Enfermagem de seu atual modelo tecnicista. Propõe o domínio sobre tecnologia e processos do cuidado sobre um eixo com ênfase mais altruísta, social e espiritual $^{(7)}$.

Assim, a Teoria do Cuidado Transpessoal não menospreza e nem deixa de reconhecer a necessidade do conhecimento técnico-científico para o cuidado ao paciente. Complementa e amplia o aspecto social e espiritual do paciente: não se encerra somente nele, mas também leva a um autoconhecimento do próprio profissional de enfermagem, ajudando tanto ao paciente como ao enfermeiro ${ }^{(7)}$.

Os focos da Teoria são: enfermagem, caracterizada por cuidar como um imperativo ético e moral de relação transpessoal, as pessoas tidas como um 
todo, isto é, seres com mentes, emoções e corpo (como sujeito, tempo e espaço); a saúde, vista experiência subjetiva de unidade e harmonia de mente, corpo e espírito associados; e meio ambiente a cujas atitudes de cuidar podem ser transmitidas ${ }^{(7)}$.

Os círculos concêntricos de cuidado também apresentam destaque. Estes são definidos a partir da a assistência focada em vários níveis interligados: pessoal, outros indivíduos, comunidade, mundo, planeta Terra e universo. O processo de cura só pode ser alcançado quando o cuidado em saúde tange esses níveis. De outro modo, a doença permanece, pois é a reciprocidade existente entre o enfermeiro e o paciente, bem como e o respeito a autonomia e liberdade de escolha deste, que auxiliam na tomada de decisões e no planejamento de seu cuidado ${ }^{(9)}$.

\section{Descrição dos componentes estruturais da teoria (conceitos, pressupostos e proposições)}

A Teoria do Cuidado Transpessoal apresenta 10 (dez) fatores de cuidado, os quais são a base para o cuidado transpessoal em sua visão holística. Abordam o ser humano como um todo biológico social e espiritual unido, que não pode ser fragmentado, e leva em consideração o profissional de enfermagem como ser humano. Nessa interação paciente/profisssional, é que se dá o processo de cuidado, cerne fundamental da enfermagem ${ }^{(10-11)}$

Esses fatores são: 1) formação de um sistema de valores humanístico-altruísta; 2) estimulação da fé-esperança; 3 ) cultivo da sensibilidade para si e para os outros; 4) desenvolvimento do relacionamento de ajuda-confiança; 5) promoção e aceitação da expressão de sentimentos positivos e negativos; 6) uso sistemático do método científico de solução de problemas para tomar decisões; 7) promoção do ensino-aprendizagem interpessoal; 8) provisão de um ambiente mental, físico, sociocultural e espiritual sustentador, protetor e/ou corretivo; 9) auxílio com a gratificação das necessidades humanas; 10) aceitação das forças existenciais e fenomenológicas ${ }^{(10)}$.

Além disso, a Teoria abrange sete pressupostos sobre o cuidado, os quais o postulam como o atributo mais valioso que a Enfermagem tem para sociedade humana, embora ameaçado pelo contínuo crescimento tecnológico na área médica ${ }^{(7-10)}$.

Os pressupostos da Teoria são: 1) o cuidado pode ser efetivado, demonstrado e praticado apenas interpessoalmente; 2) consiste de fatores que resultam na satisfação de determinadas necessidades humanas; 3) promove a saúde e o crescimento individual e familiar; 4) as respostas do cuidado aceitam a pessoa não apenas como ela é agora, mas como ela poderá ser; 5) o ambiente de cuidado é aquele que oferece o desenvolvimento potencial, enquanto permite que a pessoa escolha a melhor ação para si, em determinado momento; 6) centralizado no cuidado e não na cura, de modo que sua prática integra o conhecimento biofísico ao comportamento humano para gerar ou promover a saúde e proporcionar atendimento aos que estão doente; 7) o cuidado é a essência da prática de enfermagem e é fundamental à Enfermagem.

Esses pressupostos filosóficos permeiam o processo de cuidado humano em enfermagem, bem como, permitem o enfoque da Enfermagem no conceito de pessoa como um ser integral ${ }^{(7)}$.

\section{Aplicabilidade do cuidado transpessoal na prática de enfermagem}

Para a Teoria do Cuidado Transpessoal, a Enfermagem é "uma ciência humana de pessoas e experiências de saúde-doença humanas que são mediadas pelas transações de cuidados profissionais, científicos, estéticos e éticos"(3:1).

Assim, o cuidado transpessoal tenta se sobrepor à valorização da tecnologia, que estima somente a cura, e procura considerar como prioridade o próprio paciente. $\mathrm{O}$ cuidado pode ser considerado sua essência, já que a enfermeira participa da assistência enquanto pessoa. Deve haver uma reciprocidade entre o profissional e o paciente, de modo a preconizar a meta de estímulo a autonomia do enfermo e buscar o seu autocontrole e autoconhecimento ${ }^{(10-11)}$.

A interação enfermeiro-paciente desenvolve relações interpessoais, nas quais cada um desempenha funções específicas. Ao enfermeiro incumbe o fornecimento de apoio e proteção, com tomada de decisão científica. Ao cliente, cabem experiências positivas responsáveis por mudanças, as quais podem levar à satisfação das necessidades humanas e ao processo de ser saudável ${ }^{(7)}$.

O cuidado pode servir como essência da Enfermagem e seu atributo mais valioso. $\mathrm{O}$ alicerce por meio dos processos de cuidado, visa fornecer auxílio às pessoas para atingir um alto grau de harmonia dentro de si, de forma a promover o autoconhecimento e a própria cura ${ }^{(11-12)}$.

A utilização da teoria em questão pode 
promover o crescimento pessoal dos pacientes e da Enfermagem, traduzido por relacionamentos interpessoais mais significativos, com a ajuda/confiança e ainda pelo sentimento de liberdade. Além disso, a Teoria de Watson pode ser utilizada no planejamento para cuidados integrais, associados ao desenvolvimento do processo de Enfermagem para a execução de intervenções para o paciente ${ }^{(1)}$.

\section{CONCLUSÃO}

Os fatores de cuidado derivam da expectativa humanística, atrelados à base de conhecimentos científicos e ao conceito de reciprocidade que deve haver entre o enfermeiro e o paciente.

A partir desta descrição da Teoria do Cuidado Transpessoal de Jean Watson foi possível constatar que esta interação é a chave da mesma. O cuidado considera as necessidades psicossociais individuais. Esse modelo detém conceitos fundamentais à Enfermagem, já que associa teorias comumente usadas no ensino da área, de modo que possa ser utilizado na prática.

$\mathrm{O}$ uso desta teoria de enfermagem enriquece a identidade profissional, uma vez que proporciona alicerce sólido a partir dos fatores de cuidado preconizados por Watson, que podem ser úteis à prática, considerando o contexto e a aplicabilidade à situação em que será utilizada.

Uma de suas contribuições está na ênfase à prática do cuidado como interpessoal, baseado em fatores que resultam na satisfação de necessidades humanas, promoção da saúde e no crescimento individual e familiar, bem como no entendimento do ambiente como favorecedor do desenvolvimento pessoal e integrado ao conhecimento biofísico e comportamental humano.

Em suma, o ensino da Teoria do Cuidado Transpessoal de Watson é válida ao crescimento como seres humanos e um ponto de reflexão para futuros profissionais da área, uma vez que vislumbra reciprocidade entre o profissional e o paciente, tendo como meta seu cuidado holístico.

\section{REFERÊNCIAS}

1. Waldow VR. Cuidado humano o resgate necessário. Porto Alegre: Sagra Luzzatto; 1999.

2. Zagonel IPS. O cuidado humano transicional na trajetória de enfermagem. Rev Latino-Am Enfermagem. 1999;7(3):25-32.
3. Watson J. Caring science as sacred science. Philadelphia: F.A. Davis; 2004.

4. Severino RJ. Metodologia do trabalho científico. São Paulo: Cortez; 2000.

5. Meleis AI. Theoretical nursing: development and progress. Philadelphia: Lippincont;1997.

6. Linard AG, Pagliuca LMF, Rodrigues MSP. Aplicando o modelo de avaliação de Meleis à teoria de Travelbee. Rev Gaúch Enferm. 2004;25(1):9-16.

7. Watson J. Nursing: human science and human care. East NorwalkCT: Appleton Century Grofts; 1985.

8. Hoover J. The personal and professional impact of undertaking an educational module on human caring. J Adv Nurs. 2002;37(1):70-86.

9. Toney AM, Alligood MR. Nursing theorists and their work St Louis: Mosby; 2002.

10. Fonseca ALN, Lacerda MR, Maftum MA. O cuidado transpessoal de enfermagem no domicílio ao portador de transtorno mental e sua família. Cogitare Enferm. 2006;11(1):7-15

11. George JB. Teorias de enfermagem: os fundamentos para a prática profissional. Porto Alegre: Artes Médicas; 2001.

12. Carvalho ZMF, Damaceno MMC. Aplicação da teoria do cuidado transpessoal em pacientes paraplégicos hospitalizados: relato de experiência. Ciênc Enf. 2003;9(2):77-94.

13. Pessoa SM, Pagliuca LMF, Damasceno MMC. Teoria do cuidado humano: análise crítica e possibilidades de aplicação a mulheres com diabetes gestacional. Rev Enferm UERJ. 2006;14(3):87-92.

14. Watson J. Transformative thinking and a caring curriculum. In: Bevis EO, Watson J. Toward a caring curriculum: a new pedagogy for nursing. New York: National League for Nursing; 1989:51-60. 